\title{
Efeitos de variações cambiais em uma abordagem de dinâmica de mudança estrutural à teoria do crescimento restrito pelo balanço de pagamentos *
}

Ricardo Azevedo Araujo **

\begin{abstract}
Resumo
Neste trabalho, mostra-se que variações once-for-all do nível da taxa de câmbio podem desempenhar um papel importante na composição setorial da economia e tal fato tem implicações importantes em termos de uma versão desagregada da Lei de Thirlwall mesmo diante do argumento da insignificância quantitativa dos movimentos de preços relativos. Mostra-se, então, que a taxa de crescimento de um país compatível com o equilíbrio no balanço de pagamentos pode ser afetada por movimentos do nível de taxas de câmbio nominal e o conceito de uma taxa de câmbio natural é introduzida.
\end{abstract}

Palavras-chave: Dinâmica de mudança estrutural; Lei de Thirlwall; Taxa de câmbio.

\begin{abstract}
The effects of exchange rate variations in a structural economic dynamic approach to the balance of payment constrained growth theory

In this paper it is shown that once-for-all variations at the level of the exchange rate can play an important role in the sectoral composition of the economy. This fact has important implications in terms of a disaggregated version of Thirlwall's law, even when the argument of the quantitative unimportance of relative price movements holds. The growth rate of a country is therefore shown to be affected by once-for-all movements at the level of nominal exchange rates and the concept of a natural exchange rate is introduced.
\end{abstract}

Keywords: Structural change dynamics; Thirwall's law; Exchange rate.

JEL O19, F12.

\section{Introdução}

Um ponto importante relacionado à derivação da lei de Thirlwall em Thirlwall (1979) é o pressuposto de que os termos reais de troca ou de taxa de câmbio real permanece constante no longo prazo citado em Lopes e Cruz (2000, p. 478). Isso significa que os preços relativos são assumidos constantes, não desempenhando papel importante no longo prazo. Para Thirlwall (1979, p. 50), a irrelevância dinâmica dos preços relativos é atribuída à lei do preço único neoclássico, ou seja, o preço de um

\footnotetext{
* Trabalho recebido em 11 de maio de 2011 e aprovado em 26 de agosto de 2011. Foi feito como parte do projeto Cátedras para o Desenvolvimento do Ipea e também se beneficiou de projeto de pesquisa junto ao CNPq.

** Professor do Departamento de Economia, FACE da Universidade de Brasília, DF, Brasil. E-mail: $<$ rsaaraujo@unb.br $>$. Gostaria de agradecer, sem comprometer, a Gilberto Tadeu Lima, Mark Setterfield e aos participantes do Workshop: "Thirlwall's law and balance-of-payments constrained growth" realizado na Faculdade de Economia, Universidade de Coimbra, Portugal, 24-25 de junho de 2011, por importantes sugestões.
} 
produto deve ser o mesmo em todos os mercados, depois de considerar os custos de transporte e tarifas ${ }^{1}$. Segundo essa visão, os movimentos na taxa de câmbio nominal garantem que a lei do preço único seja válida e consequentemente, os preços relativos permanecem invariantes no longo prazo.

Alonso e Garcimartin (1998-99, p. 260) consideram que a suposição de que "os preços não importam na determinação da renda de equilíbrio não é necessária, nem suficiente para afirmar que o crescimento é limitado pela balança de pagamentos". Eles concluem que os preços relativos não desempenham qualquer função por duas razões: a primeira é apoiada por estudos empíricos que mostram que Paridade de Poder de Compra (PPP daqui por diante) vale no longo prazo $^{2}$. O segundo sugere que as elasticidades-preço são muito pequenas. Estes pontos de vista foram contestados por Leon-Ledesma (1999) para quem a existência da equalização dinâmica deve-se à fixação dos preços internacionais em mercados oligopolistas onde a diferenciação de produto é a estratégia empresarial dominante. Isso implica que os preços desempenham um papel menor na concorrência internacional e têm, portanto, pouca variação ao longo do tempo.

A partir da citada exposição, é possível concluir que, embora alguma controvérsia menor na literatura existente sobre os mecanismos responsáveis pela equalização dinâmica dos preços relativo, parece haver um consenso das diferenças observadas nas elasticidades-renda da demanda por exportações e importações, refletindo características não preço dos bens. É o importante para explicar as diferenças nas taxas de crescimento de longo prazo, com quase nenhum espaço para o papel desempenhado pelos preços relativos. Há, porém, ao menos uma razão para questionar tal ponto de vista: como apontado por Frenkel e Taylor (2006), variações temporárias, mas prolongadas na taxa de câmbio real podem ter um impacto permanente sobre a estrutura da economia e, assim, determinar as taxas de crescimento econômico no longo prazo. Em tal sentido, Barbosa-Filho (2006) conclui que o nível da taxa de câmbio real pode ser um determinante importante das elasticidades preço e renda dos fluxos comerciais, uma vez que afeta o preço relativo entre a produção de bens comercializáveis e não comercializáveis e, assim, ela pode afetar a estrutura e a taxa de crescimento da economia.

Essa visão é apoiada também por Rodrik (2008, p. 366). O autor considera que um aumento na taxa de câmbio, também visto como o preço relativo dos

(1) Mais tarde, McCombie e Thirlwall (1994, p. 307) criticaram essa visão, alegando que "[w] hen the law of one price is imposed; it is as though the whole world is producing the same good: the rationale for trade in these circumstances is unclear." Além disso, eles considera que a imposição dessa lei é especialmente questionável em um modelo sem lado da oferta, como o caso do modelo de Thirlwall.

(2) De acordo com a hipótese de PPP, a taxa de câmbio entre duas moedas irá mover-se em linha com níveis de preços relativos nas duas economias. Para commodities em que a Lei de um preço mantém o PPP, iria realizar continuamente. Neste trabalho, pretende-se mostrar que o papel real de câmbio não desempenha um papel passivo assumido nos modelos da tradição de Thirlwall. 
bens comercializáveis em termos de não comercializáveis, produz mudanças estruturais as quais podem explicar algumas experiências bem sucedidas de crescimento econômico. Se medido por sua participação no PIB ou no mercado de trabalho, o tamanho relativo da indústria depende forte e positivamente do grau de desvalorização ${ }^{3}$. Blecker $(2002$, p. 70) também confirma a posição por considerar que "a key variable in this process is the exchange rate: countries with low, competitive rates will succeed, while those with high, overvalued rates will lose out."

Porcile e Lima (2010, p. 1020) também enfatizam a importância de manterse um nível de taxa real de câmbio competitiva para estimular as exportações e promover o crescimento no longo prazo. De acordo com eles,

[c]ountries that sustained very high levels of economic growth over decades, like Korea, Taiwan, Singapore and more recently China, kept their real exchange rate at competitive levels. (...) Inversely, countries that overvalued their currency were frequently caught in low-growth traps, suffering from long periods of feeble growth.

Na verdade, alguns autores, escrevendo no contexto da lei de Thirlwall, já reconheceram que movimentos da taxa de câmbio podem afetar as taxas de crescimento. McCombie e Thirlwall (1994, p. 445), por exemplo, consideram a possibilidade de que uma desvalorização aumente a taxa de crescimento, mas, de acordo com eles, esse efeito é sentido somente sob uma depreciação contínua real e não apenas com uma desvalorização once-for-all. Ademais, outro requisito para um efeito positivo da desvalorização permanente é o cumprimento da condição de Marshall-Lerner, o qualquer que a soma das elasticidades-preço seja maior do que um. Se essa desigualdade não se verificar, mesmo desvalorizações permanentes no modelo de Thirlwall não terão efeito algum sobre as taxas de crescimento. Atesoglu (1993) encontrou um impacto quantitativamente pequeno, mas significativo dos preços relativos na taxa de crescimento das importações. Pela exclusão dos termos de troca, a elasticidade-renda da demanda não é consideravelmente afetada por movimentos de preços relativos a qual suporte adicional para a lei de Thirlwall agregados.

Nesse estágio, é importante notar que uma das principais críticas pósKeynesianas contra o modelo Neoclássico é que o último agrega toda a economia em um setor, tornando assim impossível a tarefa de realizar-se uma análise de mudança estrutural. Ademais, implícitos na representação Neoclássica, há uma definição bem conhecida e rigorosa de crescimento equilibrado, assumindo ser ele não inflacionário, com plena capacidade de utilização e que todos os setores crescem em

(3) De acordo com Rodrik (2008, p. 366), "[w] hy overvaluation is so consistently associated with slow growth is not always theorized explicitly, but most accounts link it to macroeconomic instability". No tratamento atual, é apresentado um novo tratamento teórico que liga supervalorização para o crescimento econômico por meio do efeito da primeira sobre a dinâmica estrutural da economia. 
igual proporção. A lei de Thirlwall está sujeita, porém, a essa mesma crítica já que considera as economias nacionais no agregado. É até possível dizer que uma grande quantidade de pontos levantados na literatura relacionada com a lei de Thirlwall tem alguma ligação implícita ou explícita com a hipótese de agregação ${ }^{4}$.

Hieke (1997), por exemplo, ao estudar a evidência para a economia dos EUA, encontrou uma evidência favorável para a lei de Thirlwall apenas diante da possibilidade de variações na elasticidade-renda da demanda de um período para outro. No presente trabalho, tal possibilidade é respeitada considerando-se a elasticidade-renda da demanda como média das elasticidades setoriais ponderadas pela participação de cada setor de bens comercializáveis na balança comercial. Daí, os movimentos na elasticidade da demanda agregada são devido a mudanças estruturais que têm impacto sobre a participação de cada setor. Outros autores como Alonso e Garcimantin (1998-99, p. 266) consideram que as funções de comércio não podem ser adequadamente definidas para países em que há experiência de mudanças estruturais significativas. De acordo com os citados autores, "[n] ot taking these two variables into consideration - prices and structural change, proxied by technology - may confirm Thirlwall's Law erroneously."

Araujo e Lima (2007) mostraram que de fato a dinâmica estrutural da economia pode ser transmitida por meio de funções de comércio as quais permitem coeficientes de variar de acordo com a evolução dinâmica de preferências e de progresso tecnológico ${ }^{5}$. Assim, a existência de funções comerciais estáveis não é incompatível com mudanças estruturais. No presente trabalho, é uma linha de pesquisa perseguida, mostrando-se que os preços relativos podem desempenhar um papel importante para explicar as diferenças nas taxas de crescimento de longo prazo, mesmo no contexto de PPP. Em outras palavras, mostra-se que movimentos na taxa de câmbio realmente importam na determinação da composição setorial da economia e tal fato tem implicações importantes em termos de uma versão desagregada da lei de Thirlwall. De acordo com essa visão, mudanças no nível da taxa de câmbio levam a mudanças na composição da pauta exportadora, afetando a elasticidade média das exportações.

A fim de realizar o estudo, a análise em questão parte da extensão de Araujo e Lima (2007) do modelo de mudança estrutural, considerando uma versão

(4) Alonso e Garcimartin (1998-99, p. 259) enfatizam os necessitados de se considerar uma economia com mais de um setor e graus particulares de retornos de escala para cada setor, lembrando que a demanda desempenha um papel crucial nesse contexto.

(5) Na verdade, a ideia de que a mudança estrutural pode afetar as elasticidades-renda das importações e exportações e, portanto, a taxa de crescimento tem sido em torno de algum tempo. Ver Thirlwall (1997) e Setterfield (1997). McCombie e Roberts (2002) também consideram a possibilidade de que a relação entre a elasticidade-renda da demanda por exportações para a elasticidade-renda da demanda por importações refere-se ao crescimento anterior de uma forma de U invertido. Ver Araujo e Lima (2007). 
multissetorial de lei de Thirlwall ${ }^{6}$. A partir dessa análise, é possível então mostrar que a composição setorial de uma economia pode ser afetada fortemente por movimentos once-for-all da taxa de câmbio. O conjunto de elasticidades relevantes que afetam a taxa de crescimento por meio da versão multissetorial lei de Thirlwall é, então, afetado pela competitividade dos setores. Competitividade determinada, em última análise, pelos movimentos da taxa de câmbio. Nesse sentido, mesmo quando as elasticidades setoriais da demanda por importações e exportações são constantes, pode haver mudanças estruturais devido a variações na taxa de câmbio que podem explicar a taxa de crescimento da produção. Seguindo essa abordagem, é possível destacar a relevância da lei de Thirlwall para as economias desagregadas, confirmando a visão original de que a hipótese de Crescimento Restrito pelo Balanço de Pagamentos vale para economias multissetoriais.

Para fechar o artigo, seguindo a tradição pasinettiana, introduz-se o conceito de taxa de câmbio natural que, se adotada, contribuirá com outras variáveis econômicas naturais tais como preços, taxa de lucro etc. para manter a economia em equilíbrio. Com tal conceito, transmite-se a ideia de que, embora não seja possível estabelecer a correta ou melhor taxa de câmbio, o referente não só ao equilíbrio do balanço de pagamento, mas também ao equilíbrio de outras variáveis macroeconômicas, como a demanda efetiva e o nível de emprego, devem ser prosseguidos. Assim se reconhece que, apesar de o equilíbrio ser um resultado desejável, o conceito de uma taxa de câmbio natural é apenas um critério normativo com desequilíbrios como o resultado inevitável da dinâmica econômica estrutural.

Este artigo está estruturado da seguinte forma: na próxima seção, há digressão sobre a taxa de câmbio do modelo de Pasinetti. A seção 2 estuda o impacto das variações da taxa de câmbio sobre a estrutura da economia e considera seus impactos sobre a determinação da taxa de crescimento via lei de Thirlwall. Seção 3 estabelece a noção de uma taxa de câmbio natural e a última seção resume os resultados.

\section{A taxa de câmbio no modelo Pasinettiano}

Seguindo Pasinetti (1981, 1993), supõe-se a existência de $n-1$ setores que produzem bens tradables tanto no país A como em U e não haver especialização completa. Por uma questão de conveniência, considera-se que só há bens transacionáveis nessas economia, uma vez que não se pretende comparar a dinâmica de preços entre tradables e non-tradables. De acordo com Pasinetti, o preço de cada bem em cada país é dado por:

$$
\begin{aligned}
& p_{i}^{U}=l_{i}^{U} w^{U}, \quad i=1, \ldots, n-1 \\
& p_{i}^{A}=l_{i}^{A} w^{A}, i=1, \ldots, n-1
\end{aligned}
$$

(6) Golvea e Lima (2009) forneceram suporte empírico para a versão desagregada da lei de Thirlwall. 


\section{Ricardo Azevedo Araujo}

A taxa de câmbio real é definida como:

$$
E_{U / A}=q_{U / A}\left(\frac{p_{U}}{p_{A}}\right)
$$

em que $q_{U / A}$

representa a taxa de câmbio nominal e os níveis de preços nos países $A$ e $U$ são denotados respectivamente por:

$$
\begin{aligned}
& p_{A}=\sum_{i=1}^{n-1} \theta_{i}^{A} p_{i}^{A} \\
& p_{U}=\sum_{i=1}^{n-1} \theta_{i}^{U} p_{i}^{U}
\end{aligned}
$$

em que $\theta_{i}^{U}=\left(c_{i}^{U h}+\xi c_{i}^{U e}\right) l_{i}^{U}$ é a participação do i-ésimo setor na renda nacional do país $U$ e $\theta_{i}^{A}=\left(c_{i}^{A h}+\frac{1}{\xi} c_{i}^{A e}\right) l_{i}^{A}$ é a participação do i-ésimo setor na renda nacional do país $A . c_{i}^{A e} e c_{i}^{U e}$ representam a demanda externa pelo bem $i$, mas a primeira refere-se à demanda do $A$ e a última do país $U . c_{i}^{A h} e c_{i}^{U h}$ são coeficientes de demanda domésticos para o bem $i$ nos países $A$ e $U$ respectivamente. Os $n-1$ coeficientes técnicos em cada país são denotados por $l_{i}^{A} e l_{i}^{U}$.

Mostra-se, então, que a composição setorial da economia é afetada não só pela coeficientes técnicos e demanda, mas também por movimentos na taxa de câmbio real. Para fixar as idéias, considera-se uma apreciação levando a uma taxa de câmbio sobrevalorizada. O argumento é facilmente adaptado para o caso de uma desvalorização. Não é difícil aceitar que uma taxa de câmbio sobrevalorizada afete a capacidade de exportar para alguns setores e isso pode ter um impacto não só sobre a estrutura da economia, mas também sobre a sua taxa de crescimento. A fim de verificar esse ponto, é necessário um exemplo numérico. Assume-se,então, por uma questão de conveniência apenas, que a taxa de câmbio nominal seja igual a um, isto é, $q_{U / A}=1$ e que haja apenas quarto setores na nossa economia.

Assume-se, também, que a produtividade média da economia A seja o dobro da produtividade média da economia $\mathrm{U}$, ou seja: $\sum_{i=1}^{4} l_{i}^{U}=2 \sum_{i=1}^{4} l_{i}^{A}$. O salário, portanto, real na economia A deve ser o dobro do salário real na economia $U$, ou seja, $w^{A}=2 w^{U}$.

A fim de fixar as ideias, considera-se que os coeficientes de trabalho ao longo dos quarto setores tenham a seguinte relação: $l_{1}^{U}=1.6 l_{1}^{A}, l_{2}^{U}=1.8 l_{2}^{A} \mathrm{e}$ 
$l_{3}^{U}=2.2 l_{3}^{A}, l_{4}^{U}=2.4 l_{4}^{A}$. Araujo e Teixeira (2004) mostraram mercadorias cujas diferenças de produtividade estão abaixo do dobro, ou seja, $l_{i}^{U}<2 l_{i}^{A}$, o preço será mais baixo no país $U$ que em $A$. As mercadorias que tiverem diferencial de produtividade maior do que o dobro, isto é, $l_{i}^{U}>2 l_{i}^{A}$ o preço será mais alto no país U do que no país A. Desse modo, tem-se a seguinte relação entre preços dada por $p_{1}^{U}=0.6 p_{1}^{A}, \quad p_{2}^{U}=0.8 p_{2}^{A}, \quad p_{3}^{U}=1.2 p_{3}^{A}$ e $p_{4}^{U}=1.4 p_{4}^{A}$. Não é difícil provar esse resultado. A partir das expressões (1) e (2) respectivamente, escreve-se $w^{U}=\frac{p_{i}^{U}}{l_{i}^{U}}$ e $w^{A}=\frac{p_{i}^{A}}{l_{i}^{A}}$. Como se assume que $w^{A}=2 w^{U}$, então a relação entre $p_{i}^{U}$ e $p_{i}^{A}$ será dada pela relação entre $l_{i}^{U}$ e $l_{i}^{A}$.

Esse fato mostra que o país U, apesar de ter uma menor produtividade em todos os setores, tem vantagem comparativa - de acordo com Ricardo (1921) [1817] - nos setores 1 e 2 e tende a especializar-se neles. O país $A$ tem vantagem comparativa nos setores 3 e 4 e tende a especializar-se nesses setores. Assume-se agora haver variação na taxa de câmbio nominal. É preciso considerar uma apreciação, levando a uma nova relação entre as moedas de $q_{U / A}=1.2$. Nesse caso, a relação entre os salários nominais será dado por $w^{A}=1.67 w^{U}$ apesar do fato de a produtividade média no país A ser duas vezes maior do que no país U. Assim, a relação entre preços em cada um dos setores será dada por $p_{1}^{U}=0.96 p_{1}^{A}$, $p_{2}^{U}=1.075 p_{2}^{A}, p_{3}^{U}=1.33 p_{3}^{A}, p_{4}^{U}=1.44 p_{4}^{A}$. Significa que o país U perde sua vantagem comparativa no setor 2 devido à apreciação em sua taxa de câmbio. Como no modelo apresentado o equilíbrio no balanço de pagamentos coincide com o equilíbrio no balanço comercial, é esperado que o choque na taxa de câmbio possa ser compensado pela perda de capacidade de exportação do país U. Nesse caso, a longo prazo, a taxa de câmbio nominal tende a retornar à sua posição inicial. Durante o período de transição, é possível haver mudanças estruturais na economia que pode desempenhar um papel importante na determinação da taxa de crescimento de longo prazo. No curto prazo, uma forma possível de avaliar os efeitos das variações no nível da taxa de câmbio nominal é comparar a renda per capita antes e depois da variação. Araújo e Teixeira (2004) mostraram que a renda per capita em uma versão aberta do modelo de Pasinetti pode ser escrita como:

$$
y_{U}=\sum_{i=1}^{n-1} l_{i}^{U}\left(c_{i}^{U h}+\xi c_{i}^{U e}\right)
$$

No contexto do exemplo numérico antes da apreciação cambial, a expressão (6) pode ser escrita como:

$$
y_{U}=l_{1}^{U}\left(c_{1}^{U h}+\xi c_{1}^{U e}\right)+l_{2}^{U}\left(c_{2}^{U h}+\xi c_{2}^{U e}\right)+l_{3}^{U} c_{3}^{U h}+l_{4}^{U} c_{4}^{U h}
$$


Após a apreciação cambial, a expressão (6) torna-se:

$$
y_{U}=l_{1}^{U}\left(c_{1}^{U h}+\xi c_{1}^{U e}\right)+l_{2}^{U} c_{2}^{U h}+l_{3}^{U} c_{3}^{U h}+l_{4}^{U} c_{4}^{U h}
$$

É razoável supor que os coeficientes de trabalho não sejam afetados pela variação na taxa de câmbio. Como a magnitude dos coeficientes de demanda que permanecem na expressão (6) é o mesmo daqueles que entram expressão (6), podemos concluir que a renda per capita, após a variação na taxa de câmbio é menor do que antes. Obviamente, tal análise não está levando em consideração que o efeito das mudanças na taxa de câmbio não se restringem a eliminar um dos coeficientes de exportação de expressão (6). Os coeficientes que permanecem na expressão também podem ser afetados pela mudança apontada. A insignificância quantitativa de movimentos de preços relativos pode, no entanto, ser invocada para justificar a constância dos coeficientes acima e isso nos permite concluir que o país U estará pior após a apreciação da taxa de câmbio nominal. A evidência estatística sugere, contudo, que essa suposição seja apoiada pela condição de Marshall-Lerner, sendo satisfeita para a maioria dos principais países somente no longo prazo (Kenen,1994).

Um ponto importante a considerar aqui é quando o país perde U sua vantagem comparativa no setor 2 , ele pode até enfrentar um déficit em sua balança de pagamento, implicando uma desvalorização da taxa de câmbio no longo prazo. $\mathrm{O}$ fato é particularmente verdadeiro se não houver fluxos de capital, mas na presença deles o déficit na balança comercial pode ser financiado por entradas de capital que impedem a volta da taxa de câmbio à sua posição original.

Outro ponto a ser levado em consideração é o fato de aqui ter sido assumido que o número de bens comercializáveis é dado e não é afetado por movimentos na taxa de câmbio. Existem possibilidades de que os movimentos desfavoráveis da taxa de câmbio podem induzir alguns setores a pararem de produzir bens comercializáveis e começar a produzir os não comercializáveis. Assim, os setores comercializáveis estão mais propensos a sofrer falhas de mercado que abundam nos mercados subdesenvolvidos e uma depreciação real seria promover a expansão da capacidade nesses setores, tendo aumento do crescimento. Significa que os movimentos permanentes na taxa de câmbio podem ter impacto importante sobre a estrutura econômica, por afetar a capacidade de exportação de alguns setores. (Rodrik, 2008, p. 389).

\section{Efeitos de variações no nível da taxa de câmbio nominal sobre a taxa de crescimento econômico}

A fim de avaliar o impacto de variações na taxa de câmbio sobre a taxa de crescimento, é preciso considerar, em primeiro lugar, a contribuição original de Thilwall (1979). De acordo com tal abordagem, o efeito é sentido somente sob uma depreciação contínua real e não uma variação once-for-all. Ademais, outro requisito para um efeito positivo da desvalorização permanente é o cumprimento da condição 
de Marshall-Lerner, que afirma ser a soma das elasticidades-preço maior do que um. A fim de abordar esse ponto, é necessário considerar exportação padrão e funções de importação:

$$
\begin{aligned}
& X=\left(\frac{P_{d}}{E P_{f}}\right)^{\tau} Z^{\zeta} \\
& M=\left(\frac{E P_{f}}{P_{d}}\right)^{\psi} Y^{\varphi}
\end{aligned}
$$

(8) em que $X$ representa

as exportações, $Z$ é a renda internacional, $M$ representa as importações e $Y$ é a renda doméstica. $P_{d}$ representa os preços domésticos, $P_{f}$ representa o preço externo e $E$ é a taxa de câmbio nominal. As elasticidades-preço da demanda por exportações e importações são denotadas por $\tau$ e $\psi$ e as elasticidades-renda de exportações e importações são dadas por $\zeta$ e $\varphi$, respectivamente. Em equilíbrio:

$$
P_{d} X=E P_{f} M
$$

Tomando logaritmos e diferenciando as expressões (7), (8) e (9), há obtenção de:

$$
\begin{gathered}
\hat{x}=\tau\left(\hat{p}_{d}-\hat{p}_{f}-\hat{e}\right)+\zeta \hat{z} \\
\hat{m}=\psi\left(\hat{p}_{f}+\hat{e}-\hat{p}_{d}\right)+\varphi \hat{y} \\
\hat{p}_{d}+\hat{x}=\hat{p}_{f}+\hat{e}+\hat{m}
\end{gathered}
$$

em que os chapéus denotam as taxas de crescimento. Substituindo (7)' e (8)' na expressão (9)' obtém-se:

$$
\hat{y}=\frac{(1+\tau+\psi)\left(\hat{p}_{d}-\hat{p}_{f}-\hat{e}\right)+\zeta \hat{z}}{\varphi}
$$

De acordo com a expressão (10), apenas uma desvalorização contínua $-\hat{e}>0$ - irá melhorar a taxa de crescimento compatível com o equilíbrio de longo prazo no balanço de pagamentos. Assim acontecerá, se a soma das elasticidades da demanda por importações e exportações for maior do que em valor absoluto que é a condição de Marshall-Lerner: $|\tau+\psi|>1$. Uma desvalorização once-for-all da taxa de câmbio, todavia, não afetará o equilíbrio do balanço de pagamentos no longo prazo ${ }^{7}$ (McCombie; Thirlwall, 1994, p. 236).

(7) Sob a condição de Marshall- Lerner é possível obter o que Perraton (2003) denominou a forma forte da hipótese BPCG, $\hat{y}=\frac{\zeta}{\varphi} \hat{z}$. Considerando que vale a PPC, a Lei de Thirlwall pode ser rescrita como: $\hat{y}=\frac{1}{\varphi} \hat{x}$ que ele denomina a forma fraca da hipótese BPCG. 
De acordo com essa visão, choques temporários na taxa de câmbio real podem ter efeitos transitórios e não exercem qualquer efeito sobre a estrutura subjacente da economia. Se os custos de entrada no mercado, no entanto, afundarem, os choques de taxas de câmbio podem alterar a estrutura do mercado doméstico e, assim, ter efeitos reais persistentes. Nesse caso, a taxa de crescimento compatível com o equilíbrio no balanço de pagamento pode ser afetada pela mudança na estrutura como sustentam Araujo e Lima (2007). Pode-se argumentar que os efeitos de um choque temporário da taxa de câmbio serão compensados para manter o equilíbrio intertemporal do balanço de pagamentos. Assim, uma valorização temporária será automaticamente seguida por uma desvalorização que irá restaurar o valor inicial da taxa de câmbio e não haverá efeito sobre a dinâmica estrutural, mas essa não é toda a história. De acordo com Baldwin (1988), se os custos de entrada no mercado são irrecuperáveis, choques suficientemente grandes na taxa de câmbio real podem alterar a estrutura do mercado doméstico e, assim, induzir a histerese. No caso, mesmo choques temporários na taxa de câmbio podem resultar em histerese dos preços de importação e as quantidades. Opinião também confirmada por Baldwin e Krugman (1989) segundo a qual as flutuações da taxa de câmbio provocam decisões de entrada ou saída e delas não são revertidas quando a moeda retorna a seu nível anterior.

Seguindo esse raciocínio, mostra-se a seguir, por meio da versão desagregada da lei de Thirlwall, que mesmo uma variação once-for-all na taxa de câmbio terá impacto sobre a taxa de crescimento compatível com o equilíbrio no balanço de pagamentos. Na forma forte da hipótese BPCG, a lei Thirlwall multissetorial citada em Araujo e Lima (2007) pode ser escrito como:

$$
\sigma_{y}^{U}=\frac{\sum_{i=1}^{n-1} \xi \beta_{i} c_{i}^{U e} l_{i}^{U}}{\sum_{i=1}^{n-1} \phi_{i} c_{i}^{A e} l_{i}^{U}} \sigma_{y}^{A}
$$

(11) em que $\frac{\dot{y}_{A}}{y_{A}}=\sigma_{y}^{A}$,

$\frac{\dot{y}_{U}}{y_{U}}=\sigma_{y}^{U}, \phi_{i}$ é a elasticidade renda por importações e $\beta_{i}$ é a elasticidade renda das exportações associada ao bem $i$. A fim de considerar o efeito dos movimentos da taxa de câmbio sobre a expressão (11) ocorre, seguindo a seção anterior,uma apreciação na taxa de câmbio. O objetivo da análise é mostrar que, mesmo nesse caso, a estrutura da economia poderá ser alterada devido a variações na composição das exportações e isso terá impacto sobre a taxa de crescimento de acordo com a lógica da lei de Thirlwall desagregada.

Assim, no primeiro cenário, o país U exporta bens 1 e 2, enquanto importa os bens 3 e 4 . Quando ocorre a valorização, esse país perde inicialmente sua vantagem comparativa em termos do bem 2 e passa a não exportar mais esse bem, mas mantém 
suas exportações de bem 1. Quando a taxa de câmbio retorna, porém, ao valor inicial, o país U recupera sua vantagem comparativa em termos do bem 2 , mas agora a participação desse bem no total das exportações será diferente de seu valor inicial. Nesse caso, a lei de Thirlwall em cada um dos cenários é dada por:

$$
\begin{gathered}
\sigma_{y b}^{U}=\frac{\beta_{1} c_{1 b}^{U e} l_{1 b}+\beta_{2} c_{2 b}^{U e} l_{2 b}}{\phi_{3} c_{3 b}^{A e} l_{3 b}+\phi_{4} c_{4 b}^{A e} l_{4 b}} \sigma_{y}^{A} \\
\sigma_{y a}^{U}=\frac{\beta_{1} c_{1 a}^{U e} l_{1 a}+\beta_{2} c_{2 a}^{U e} l_{2 a}}{\phi_{3} c_{3 a}^{A e} l_{3 a}+\phi_{4} c_{4 a}^{A e} l_{4 a}} \sigma_{y}^{A}
\end{gathered}
$$

A fim de comparar as taxas de crescimento em expressões (12) e (13), supõese, sem perda de generalidade, que $\beta_{2}>\beta_{1}$. Essa hipótese tem como objetivo levar adiante a ideia de que uma apreciação da taxa de câmbio leva o país U a perder sua vantagem comparativa em termos do bem com renda mais alta de elasticidade da demanda. Se não se estivesse assumindo que a taxa de câmbio irá retornar ao nível anterior, seria razoável supor que o coeficiente desaparece na expressão (12) uma vez que o coeficiente de exportação será igual a zero, o que certamente irá gerar uma menor taxa de crescimento para o país U após a apreciação. Como estamos supondo que a taxa de câmbio retorna ao nível original, assume-se que o país U seja capaz de recuperar sua vantagem comparativa em termos do bem 2. Isso significa que o coeficiente será diferente de zero, mas, provavelmente, menor de que seu valor antes da apreciação cambial. É hipótese razoável por uma série de razões: primeiro, como apontado por Baldwin (1988) e Baldwin e Krugman (1989), existem custos afundados relacionados ao investimento e uma apreciação da taxa de câmbio pode ter induzido investimentos estrangeiros, impedindo assim que o coeficiente de exportação volte para o nível anterior. Segundo, a perda sofrida por empresas nacionais, devido a uma apreciação não pode ser facilmente compensada por um aumento da demanda externa.

Matematicamente, isso pode ser visto considerando-se que os numeradores de expressões (12) e (13) são a média ponderada das elasticidades setoriais. Concluise que o numerador da expressão (12), $\beta_{1} c_{1 b}^{U e} l_{1 b}+\beta_{2} c_{2 b}^{U e} l_{2 b}$ é, em geral, maior do que o numerador da expressão (13), $\beta_{1} c_{1 a}^{U e} l_{1 a}+\beta_{2} c_{2 a}^{U e} l_{2 a}$. Nota-se que em ambas as expressões tais produtos medem o peso das elasticidades setoriais antes e depois da apreciação. É razoável supor que os denominadores não mudarão substancialmente. É possível concluir, então, que a taxa de crescimento no segundo cenário é menor do que no primeiro, uma vez que o numerador é menor e o denominador não será muito diferente em ambas as expressões. No Apêndice, uma série de simulações é realizada a fim de verificar a robustez de tal raciocínio. 
Esse fato confirma a suspeita de que mesmo uma variação once-for-all na taxa de câmbio pode desempenhar um papel importante na mudança estrutural, uma vez que a competitividade dos sectores é fortemente influenciada pela taxa de câmbio nominal. A ilustração mostra que a produtividade é importante, mas não decisiva na determinação da capacidade de exportação. Ademais, é importante considerar que as elasticidades de importação e exportação, embora constantes, ainda desempenham um papel importante na determinação da taxa de crescimento do país. Para se entender de forma adequada sua participação no processo de crescimento, é necessário, porém, ponderá-las pela participação de cada setor na renda nacional, sendo essa percentagem fortemente afetada pela taxa de câmbio nominal.

Embora o trabalho não discuta as causas das variações da taxa de câmbio nominal, há ao menos uma circunstância em que a fonte de tais movimentos também pode ser afetada pelas elasticidades de produtos fabricados e exportados, caso em que os países geram superávits comerciais significativos nos setores primários exportadores. Considere, por exemplo, que o setor 2 no país U seja manufatureiro. Após a apreciação da taxa de câmbio no país, ele pode deixar de exportar esse bem e resultar em desindustrialização. Se a apreciação da taxa de câmbio deve-se a um superávit comercial significativo em produtos primários, então, o fenômeno é identificado como Doença Holandesa, o qual tem prejudicado a experiência de crescimento dos novos países industrializados que descobriram recursos naturais, visto em Palma (2005). Se o país é capaz de eliminar o efeito negativo produzido por um superávit comercial em produtos primários sobre a taxa de câmbio, então, é capaz de avançar para o processo de industrialização por meio da produção e exportação de bens industriais produzidos pelo setor 2. O processo de desindustrialização não é o resultado desejado para um país em desenvolvimento que pretende promover as mudanças estruturais adequadas que lhe permitam crescer mais rapidamente.

Como Pasinetti (1993, 1981), alguns autores enfatizam que os principais ganhos que se obtêm a partir de relações internacionais estão relacionados à aprendizagem. Aqui, consideram-se fixos os coeficientes de trabalho - devido à inexistência de progresso tecnológico - mas espera-se que quanto maior a participação de um país em atividades de exportação, maior o acesso ao conhecimento por meio da aprendizagem. Assim, uma taxa de câmbio sobrevalorizada pode danificar a capacidade de absorver a aprendizagem internacional, pois afeta a capacidade de participação no comércio internacional.

\section{A taxa natural de câmbio}

Nas seções anteriores, os efeitos das mudanças na taxa de câmbio foram estudadas, mas não se determinou qual a taxa de câmbio a ser adotada. Para muitos autores, a noção de taxa de câmbio de equilíbrio está associada ao equilíbrio no 
balanço de pagamentos ${ }^{8}$. Isso não nos dá, porém, uma informação precisa, uma vez que há diferentes valores da taxa de câmbio que geram tal equilíbrio. Ademais, há forte evidência de que taxas de câmbios desvalorizadas são propícias ao crescimento econômico, mas o resultado não é inequívoco. Taxas de câmbio sobrevalorizadas podem ter um impacto positivo no crescimento econômico por meio do barateamento do preço de bens de capital importados, permitindo assim um maior acesso de países subdesenvolvidos a técnicas de produção que requerem o uso de bens de capital específicos ${ }^{9}$. Outro efeito positivo de uma taxa de câmbio apreciada é por meio do efeito positivo sobre a taxa de lucro que pode estimular a acumulação de capital e estimular o crescimento econômico.

A tentativa, portanto, de estabelecer a correta ou a melhor taxa de câmbio com base apenas no desempenho do produto pode ser enganosa e, assim, tal tarefa deve ser substituída pelo objetivo de encontrar uma taxa de câmbio natural, isto é, compatível com uma economia natural crescendo em ritmo de pleno emprego. Esse procedimento também está de acordo com o a determinação do valor natural de outras variáveis econômicas, tais como a taxa de lucro, a taxa de juros etc. feita por Pasinetti (1981, 1993). Assim, a taxa de câmbio natural será aquela que se adotada, permite o pleno emprego e plena utilização da capacidade.

Como demonstrado por Araújo e Teixeira (2004) em um sistema dinâmico de economia aberta, a condição demanda efetiva é dividida em duas condições, ou seja, uma para o pleno emprego, outra para a despesa total da renda nacional. A razão pela qual temos duas condições ao invés de uma é clara: em uma economia aberta, a renda nacional pode ser gasta em produtos importados e, nesse caso, o cumprimento da condição do pleno gasto da renda não implica a satisfação da condição de pleno emprego. Como apontado por tais autores, quando cumpridas as duas condições simultaneamente, uma outra é automaticamente satisfeita, pois é o equilíbrio no balanço de pagamentos. A fim de explicitar esse ponto formalmente, importante considerar a condição de pleno emprego e a de pleno gasto da renda em sistema aberto e dinâmico, que são dadas por:

$$
\begin{aligned}
& \sum_{j=1}^{m}\left(c_{j}^{U h}+\xi c_{j}^{U e}\right) l_{j}=1 \\
& \sum_{j=1}^{m}\left(c_{j}^{U h}+c_{j}^{U i}\right) l_{j}=1
\end{aligned}
$$

(8) De acordo com essa visão, desalinhamentos na taxa de câmbio real implicam algum tipo de desequilíbrio macroeconômico, o que por si só já é um fator negativo para o crescimento econômico. Para um survey dessa visão, veja Berg e Miao (2010).

(9) Na verdade, países subdesenvolvidos enfrentam na maioria das vezes dependência de capital e há uma grande evidência empírica de o progresso tecnológico estar incorporado nos bens de capital. 
Por meio da equalização do lado esquerdo das duas expressões, é possível provar, após algumas manipulações algébricas que uma nova condição a ser vista como incorporando uma noção de equilíbrio na balança de pagamento é também satisfeita. Do ponto de vista do país U, ela pode ser expressa como:

$$
\sum_{j=1}^{m}\left(\xi c_{j}^{U e}-c_{j}^{U i}\right) l_{j}=0
$$

Como apontado por Araujo e Teixeira (2004),

[t]he basic intuition for this result can be grasped by observing that the labor coefficients $l_{j}$ 's are being used to weight both the export and import demand coefficients for commodity $j$. Therefore, this condition requires that exported commodities expressed in terms of quantity of labor in country $U$ be equal to the quantity of imported commodities also expressed in terms of labor in $U$.

Considerando que o coeficiente técnico do setor $j$ pode ser escrito como $l_{j}=\frac{p_{j}}{w}$, essa expressão pode ser rescrita como:

$$
\sum_{j=1}^{m}\left(\xi c_{j}^{U e}-c_{j}^{U i}\right) p_{j}=0
$$

Essa condição expressa o equilíbrio da balança comercial em termos de preços. Em uma economia do trabalho pura, há equivalência entre o equilíbrio da balança comercial em termos de preços e em termos de quantidades de trabalho. A análise acima mostra que o equilíbrio na balança de pagamento pode ser visto como o resultado do equilíbrio no mercado de trabalho e no mercado de bens. Isso não exclui a possibilidade de equilíbrio na balança de pagamento sem o pleno emprego e o pleno gasto da renda nacional. Note que se (16) é satisfeita, então o lado esquerdo das expressões (14) e (15) devem ter o mesmo valor, não necessariamente igual a um. O equilíbrio no balanço de pagamentos é, portanto, uma condição necessária, mas não suficiente para garantir o pleno emprego e o pleno gasto da renda nacional. As expressões (14) e (15) podem, todavia, ser adotadas para estabelecer um caminho de equilíbrio útil na determinação da taxa de câmbio natural.

Um importante ponto a ser considerado aqui é que a taxa de câmbio natural não irá privilegiar igualmente todos os setores: alguns deles podem estar encolhendo enquanto outros, expandindo-se. Na verdade, o resultado final da dinâmica de mudança estrutural é o desemprego estrutural e o desequilíbrio. $O$ conceito de taxa natural de câmbio, no entanto, chama a atenção para a existência de um equilíbrio normativo em que as variáveis econômicas podem ser adotadas como parâmetros para os formuladores de políticas tomarem suas decisões. 


\section{Considerações finais}

Há um consenso entre os economistas heterodoxos que o desafio do desenvolvimento econômico é de fato o desafio de se proceder às mudanças estruturais adequadas citado em Ocampo (2005). Nesse sentido, variáveis como preferências e tecnologias desempenham um papel fundamental uma vez que determinam o padrão de evolução da composição setorial da economia. Outras variáveis econômicas, porém, tais como taxa de câmbio pode desempenhar um papel importante também. Essa é a mensagem central do artigo. Mostra-se que as variações no nível da taxa de câmbio nominal afetam os benefícios obtidos a partir do princípio das vantagens comparativas. Seguindo tal raciocínio e considerando uma versão multissetorial da Lei de Thirlwall, é possível mostrar que a taxa de crescimento de um país pode ser fortemente afetada por movimentos once-for-all da taxa de câmbio nominal. Uma apreciação dessa taxa reduz a competitividade das indústrias nacionais, produzindo mudanças estruturais que podem desacelerar o crescimento e o investimento. Ademais, um país ainda pode aumentar sua taxa de crescimento, mesmo enfrentando baixas taxas de crescimento da renda mundial desde que seja capaz de mudar a composição setorial das exportações e/ou importação conforme a abordagem demonstrada aqui. $\mathrm{O}$ resultado contradiz a sabedoria convencional de que apenas movimentos permanentes e contínuos da taxa de câmbio desempenham um papel importante para o crescimento econômico no longo prazo. Assim, a visão heterodoxa que o processo de crescimento econômico nos países em desenvolvimento pode ser induzido por estratégias de crescimento é confirmada, como é visto em Thirlwall (1997). Embora a análise mostre que o manejo adequado das taxas de câmbio pode promover o crescimento econômico, não estabelece que taxa de câmbio deve ser adotada. A fim de atenuar essa lacuna, introduziu-se a noção de uma taxa de câmbio natural. Com as noções de taxa de lucro natural e taxa natural de juros natural, o conceito de uma taxa de câmbio natural dá a noção de uma taxa de câmbio que contribui para a manutenção do equilíbrio em uma economia natural. Tal análise também fornece critérios normativos para escolher a taxa de câmbio que irá produzir o pleno emprego ao longo do equilíbrio na balança de pagamento.

\section{Referências bibliográficas}

ARAUJO, R.; LIMA, G. A structural economic dynamic approach to balance of payment constrained growth. Cambridge Journal of Economics, v. 31, n. 5, p. 755-774, 2007.

; TEIXEIRA, J. A Pasinettian approach to international economic relations: the pure labour case. Review of Political Economy, v. 16, n. 1, p. 117-129, 2004.

ALONSO, J.; GARCIMARTIN, C. A new approach to balance-of-payments constraint: some empirical evidence. Journal of Post-Keynesian Economics, v. 21, n. 2, p. 259-282, 1998-99. 
ATESOGLU, H. Balance-of-payments-constrained growth model and its implications for the United States. Journal of Post-Keynesian Economics, v. 19, n. 3, p. 327-335, 1997.

- Balance-of-payments constrained growth: evidence from the United States. Journal of Post-Keynesian Economics, v. 15, n. 4, p. 507-514, 1993.

BAIRAM, E. Levels of economic development and appropriate specification of the Harrod foreign-trade multiplier. Journal of Post-Keynesian Economics, v. 19, n. 3, p. 337-343, 1997.

BALDWIN, R. Hystereses in import prices: the Beahhead effect. The American Economic Review, v. 78, n. 4, p. 773-785, 1998.

; KRUGMAN, P. Persistent trade effects of large exchange rate shocks. The Quaterly Journal of Economics, v. 104, n. 4, p. 635-654, 1989.

BARBOSA-FILHO, N. Exchange rates, growth and inflation. Paper submitted to the Annual Conference on Development and Change, Campos do Jordão, Brazil, Nov. 18-20, 2006.

BERG, A.; MIAO, Y. The real exchange rate and growth revisited: the Washington consensus strikes back? IMF Working Paper 10/58, 2010.

BLECKER, R. The balance of payment-constrained growth model and limits to exportled growth. In: DAVIDSON, P. (Ed.). A post-Keynesian perspective on twenty-first century economic problems. UK: Edward Elgar Publishing Limited, 2002.

FRENKEL, R.; TAYLOR, L. Real exchange rate, monetary policy and employment. United Nations, 2006. (DESA Working Paper, n. 19).

GOLVEA, R.; LIMA, G. Structural change, balance-of-payments constraint and economic growth: evidence from the multi-sectoral Thirlwall's law. In: BRAZILIAN MEETING OF ECONOMICS, 37, 2009. Proceedings...

GOLVEA, R.; LIMA, G. Balance-of-payments-constrained growth in a multisectoral framework: a panel data investigation. Germany: University Library of Munich, 2011. (MPRA Paper, n. 29816).

HIEKE, H. Balance-of-payment-constrained growth: a reconsideration of the evidence for the U.S. economy. Journal of Post Keynesian Economics, v. 19, n. 3, p. 313-325, 1997.

KENEN, P. Essays in international economics. Princenton; New Jersey: Princenton University Press, 1980.

LEON-LEDESMA, M. An application of Thirlwall's law to the Spanish economy. Journal of Post-Keynesian Economics, v. 21, n. 3, p. 431-439, 1999.

LOPEZ, J.; CRUZ, A. Thirlwall's law and beyond: the Latin American experience. Journal of Post Keynesian Economics, v. 22, n. 3) p. 477-495, 2000 .

McCOMBIE, J. On the empirics of balance of payments-constrained growth. Journal of Post Keynesian Economics, v. 19, n. 3, p. 345-376, 1997.

; ROBERTS, M. The role of balance of payments in economic growth. In:

SETTERFIELD, M. (Org.). The economics of demand-led growth: challenging the supply- 
side vision of the long run. Cheltenham, UK; Northampton, MA: Edward Elgar, 2002. p. $87-114$.

; THIRLWALL, A. Economic growth and balance-of-payments constraint. New York: St. Martins Press, 1994.

MORENO, J. Mexico's economic growth and the balance of payments constraint: a cointegration analysis. International Review of Applied Economics, v. 13, n. 2, p. 150-159, 1999.

OCAMPO, A. The quest for dynamic efficiency. In: OCAMPO, José A. (Ed.). Beyond reforms: structural dynamics and macroeconomic vulnerability. Stanford University Press, 2005.

PALMA, G. Four sources of 'de-industrialization' and a new concept of the 'dutch disease'. In: OCAMPO, José A. (Ed.). Beyond reforms: structural dynamics and macroeconomic vulnerability. Stanford University Press, 2005.

PASINETTI, L. Structural change and economic growth - A theoretical essay on the dynamics of the wealth of the nations. Cambridge, UK: Cambridge University Press, 1981.

- Structural economic dynamics - A theory of the economic consequences of human learning. Cambridge, UK: Cambridge University Press, 1993.

PERRATON, J. Balance of payments constrained growth and developing countries: an examination of Thirlwall's hypothesis. International Review of Applied Economics, v. 17, n. 1 , p. 1-22, 2003.

PORCILE, G.; LIMA, G. Real exchange rate and elasticity of labour supply in a balanceof-payments constrained macrodynamics. Cambridge Journal of Economics, v. 34, p. 10191039, 2010.

RICARDO, D. Principles of political economy and taxation. In: SRAFFA, Pierro (Ed.). Works and correspondences of David Ricardo. I. Cambridge: Cambridge University Press, 1921 [1817].

RODRIK, D. The real exchange rate and economic growth: comments and discussions. Brookings Papers on Economic Activity, p. 365-412, Fall 2008.

THIRLWALL, A. The balance of payments constraint as an explanation of international growth rates differences. Banca Nazionale del Lavoro Quarterly Review, 128, p. 45-53, 1979.

- Growth and development. 5th ed. London: Macmillan Press Ltd., 1994.

. Reflections on the concept of balance-of payments-constrained growth. Journal

of Post Keynesian Economics, v. 19, n. 3, p. 377-386, 1997. 


\section{Appendix: Numerical simulations}

Matlab foi adotado para realizar as simulações numéricas. Quatro setores foram considerados: matérias-primas, agricultura tropical, máquinas e produtos químicos. As estimativas para elasticidades-renda de exportação e importação foram obtidas a partir de Lima e Golvea (2011).

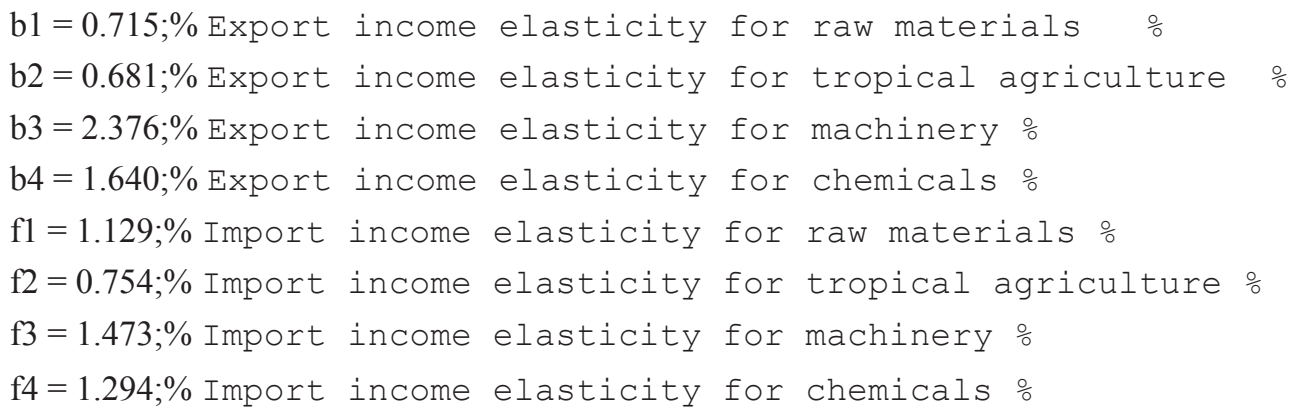

A fim de fornecer generalidade para as simulações, a maioria dos parâmetros do modelo, tais como a taxa de crescimento setorial da demanda, as de progresso tecnológico e o valor inicial para o trabalho e de exportação e coeficientes de importação foram deixados para serem escolhidos pelo computador como variáveis aleatórias. Significa que, em todos os valores de simulação dessas variáveis, eles serão atribuídos e a taxa de crescimento do país U vai ser calculada de acordo com a versão multissetorial da Lei de Thirlwall. A taxa de crescimento do país A será estabelecida como 0,02 por período. No primeiro conjunto de simulações, Gráficos 1 a 3, a taxa de crescimento do país U é calculada por cem períodos e assume-se que as mudanças estruturais na economia não são o resultado de mudanças na taxa de câmbio nominal. Na segunda, Gráficos 4-6, e terceiro, Gráficos 7-9, conjuntos de simulações supõem, no período de cinquenta, haver uma apreciação da taxa de câmbio que afeta a capacidade de exportação do país U, fazendo-o perder a vantagem comparativa na exportação de bens de consumo 2 .

Seguindo a premissa feita por Araujo e Lima (2007), nas simulações 4-6, o efeito da apreciação da taxa de câmbio sobre o coeficiente de demanda é instantânea levando-o a atingir o valor zero. No terceiro conjunto de simulações, 7-9, essa hipótese é relaxada, assumindo que, apesar de o país U perder a sua vantagem comparativa, o coeficiente de exportação não desaparece instantaneamente, mas vai a zero, seguindo uma regra exponencial. Nas tabelas a seguir, os parâmetros escolhidos de forma aleatória são apresentados por três conjuntos de simulações. Embora a taxa de crescimento do país U varie consideravelmente entre essas simulações, devido à ampla gama de variação dos parâmetros escolhidos, o comportamento da balança de pagamento de taxa de crescimento limitado é semelhante para os três set ups. As Figuras 1-3 expressam o caminho de crescimento dinâmico na ausência de movimentos na taxa de câmbio. Em 4-9, o caminho dinâmica da taxa de crescimento é analisada sob o pressuposto de que no período cinquenta há uma apreciação da taxa de câmbio que afeta a estrutura da economia como assumido na seção anterior. $\mathrm{O}$ caso em que a alteração do coeficiente de exportação é instantânea, é plotado na esquerda, enquanto o gráfico de uma adaptação gradual em direção a zero é mostrado à direita. O objetivo de colocar esses gráficos juntos é mostrar que, embora a queda na taxa de crescimento seja menor no segundo caso, essencialmente, mantém o mesmo comportamento dos gráficos do primeiro caso. 
Efeitos de variações cambiais em uma abordagem e dinâmica de mudança estrutural...

Simulação 1

\begin{tabular}{c|c|c|c}
\hline $\begin{array}{c}\text { Growth rate of } \\
\text { demand }\end{array}$ & $\begin{array}{c}\text { Technological } \\
\text { progress }\end{array}$ & $\begin{array}{c}\text { Initial values for } \\
\text { labour coefficients }\end{array}$ & $\begin{array}{c}\text { Initial values for } \\
\text { export and import } \\
\text { enefficients }\end{array}$ \\
\hline $\mathrm{rl}=5.9490 \mathrm{c}-004$ & $\rho 1=2.2175 \mathrm{c}-004$ & $11(0)=0.0855$ & $\mathrm{x} 1(0)=4.2417$ \\
$\mathrm{r} 2=2.6221 \mathrm{c}-004$ & $\rho 2=1.1742 \mathrm{c}-004$ & $12(0)=0.2625$ & $\mathrm{x} 2(0)=5.0786$ \\
$\mathrm{r} 3=6.0284 \mathrm{c}-004$ & $\rho^{3}=2.9668 \mathrm{c}-004$ & $13(0)=0.8010$ & $\mathrm{i} 3(0)=9.2885$ \\
$\mathrm{r} 4-7.1122 \mathrm{c}-004$ & $\rho 4-3.1878 \mathrm{c}-0.04$ & $14(0)=0.0292$ & $\mathrm{i} 4(0)-7.3033$ \\
\hline
\end{tabular}

Simulaçầo 2

\begin{tabular}{c|c|c|c}
\hline $\begin{array}{c}\text { Growth rate of } \\
\text { demand }\end{array}$ & $\begin{array}{c}\text { Technological } \\
\text { progress }\end{array}$ & $\begin{array}{c}\text { Initial values for } \\
\text { labour coefficients }\end{array}$ & $\begin{array}{c}\text { Initial values for } \\
\text { export and Import } \\
\text { coefficients }\end{array}$ \\
\hline $\mathrm{rl}=9.1329 \mathrm{c}-004$ & $\rho \mathrm{l}=3.3536 \mathrm{c}-004$ & $11(0)=0.4942$ & $\mathrm{xl}(0)=1.0676$ \\
$\mathrm{r}=7.9618 \mathrm{c}-004$ & $\rho 2=6.7973 \mathrm{c}-004$ & $12(0)-0.7791$ & $\mathrm{x} 2(0)-6.5376$ \\
$\mathrm{r} 3-9.8712 \mathrm{e}-005$ & $\rho 3-1.3655 \mathrm{e}-0.04$ & $13(0)-0.7150$ & $\mathrm{i} 3(0)-8.9092$ \\
$\mathrm{r} 4-2.6187 \mathrm{e}-004$ & $\rho 4-7.2123 \mathrm{e}-004$ & $14(0)-0.9037$ & $\mathrm{i} 4(0)-3.3416$ \\
\hline
\end{tabular}

Simulaçĩo 3

\begin{tabular}{c|c|c|c}
\hline $\begin{array}{c}\text { Growth rate of } \\
\text { demand }\end{array}$ & $\begin{array}{c}\text { Technological } \\
\text { progress }\end{array}$ & $\begin{array}{c}\text { Initial values for } \\
\text { labour coefficients }\end{array}$ & $\begin{array}{c}\text { Initial values for } \\
\text { export and import } \\
\text { coefficients }\end{array}$ \\
\hline $\mathrm{r} 1=4.8861 \mathrm{e}-004$ & $\rho 1-9.6309 \mathrm{e}-004$ & $11(0)-0.6791$ & $\times 1(0)-4.8890$ \\
$\mathrm{r} 2-5.7853 \mathrm{e}-004$ & $\rho 2-5.4681 \mathrm{e}-004$ & $12(0)-0.3955$ & $\times 2(0)-6.2406$ \\
$\mathrm{r} 3=2.3728 \mathrm{e}-004$ & $\rho 3=5.2114 \mathrm{e}-004$ & $13(0)=0.3674$ & $\mathrm{i} 3(0)=0.3774$ \\
$\mathrm{r} 4=4.5885 \mathrm{e}-004$ & $\rho 4=2.3159 \mathrm{e}-004$ & $14(0)=0.9880$ & $\mathrm{i} 4(0)=8.8517$ \\
\hline
\end{tabular}

Gráfico 1

Taxa de crescimento do país U - Simulação 1

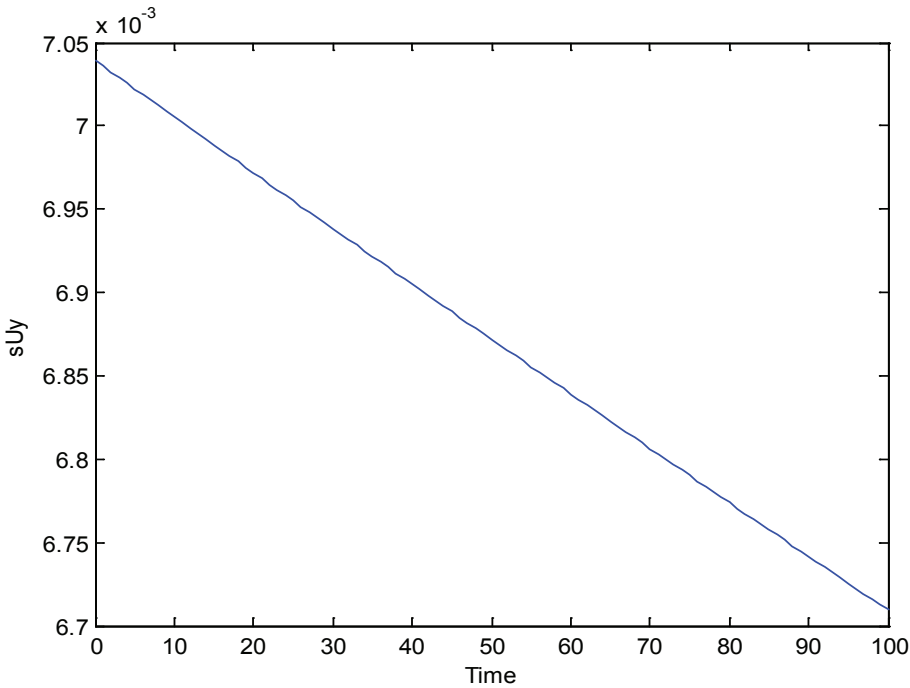


Gráfico 2

Taxa de crescimento do país U - Simulação 2

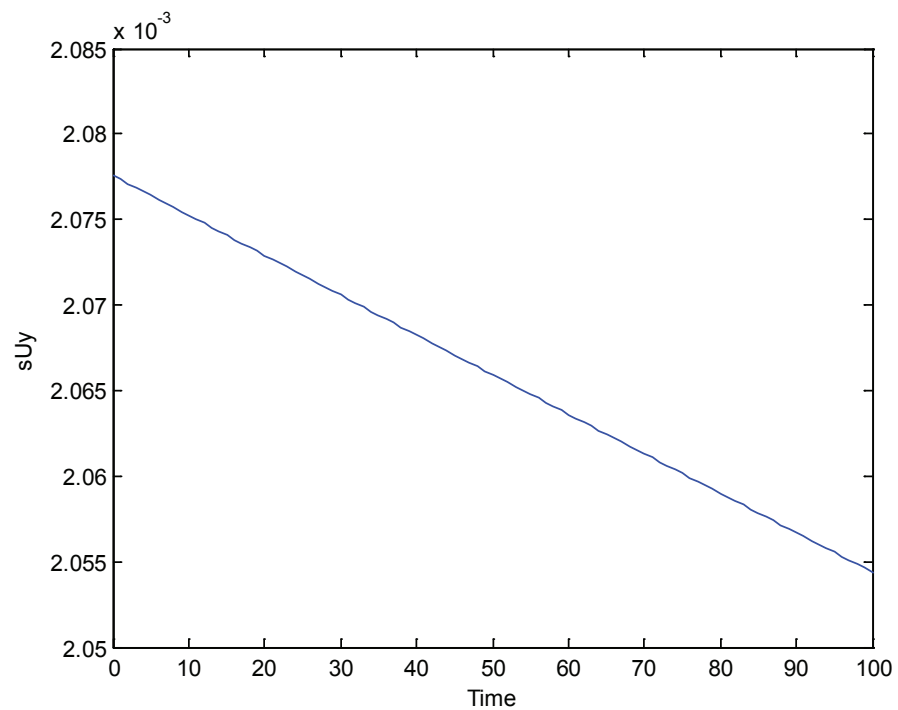

Gráfico 3

Taxa de crescimento do país U - Simulação 3

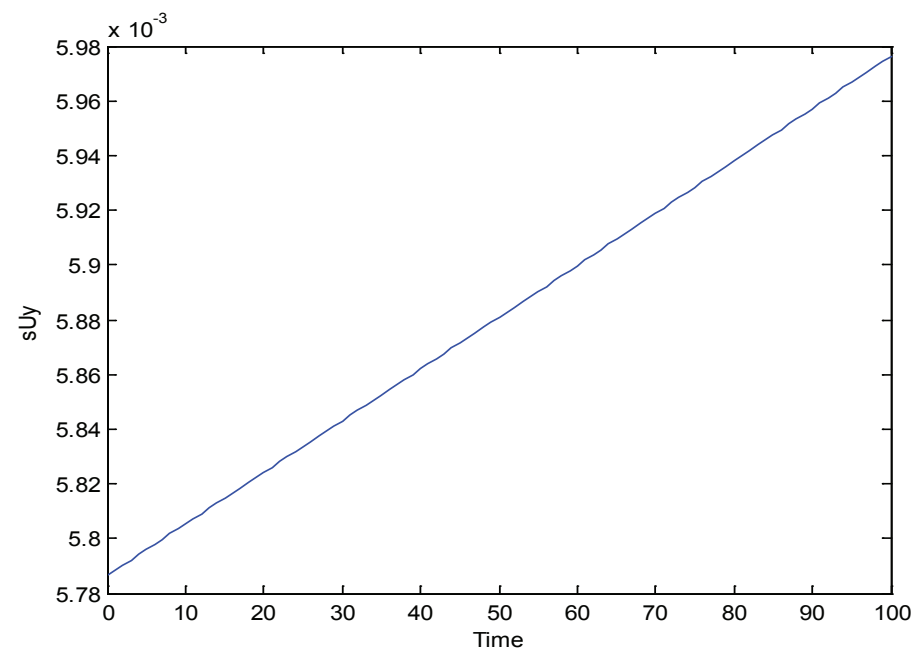


Efeitos de variações cambiais em uma abordagem e dinâmica de mudança estrutural...

Gráficos 4 e 7

Taxa de crescimento do país U antes e depois da apreciação cambial no período 50. Simulação 1
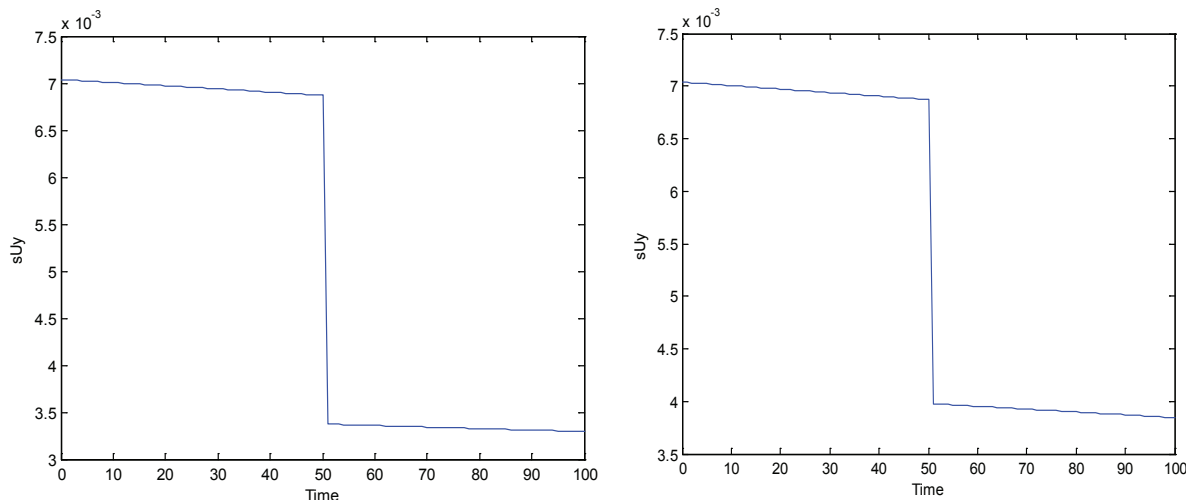

Gráfico 5 e 8

Taxa de crescimento do país U antes e depois da apreciação cambial no período 50. Simulação 2
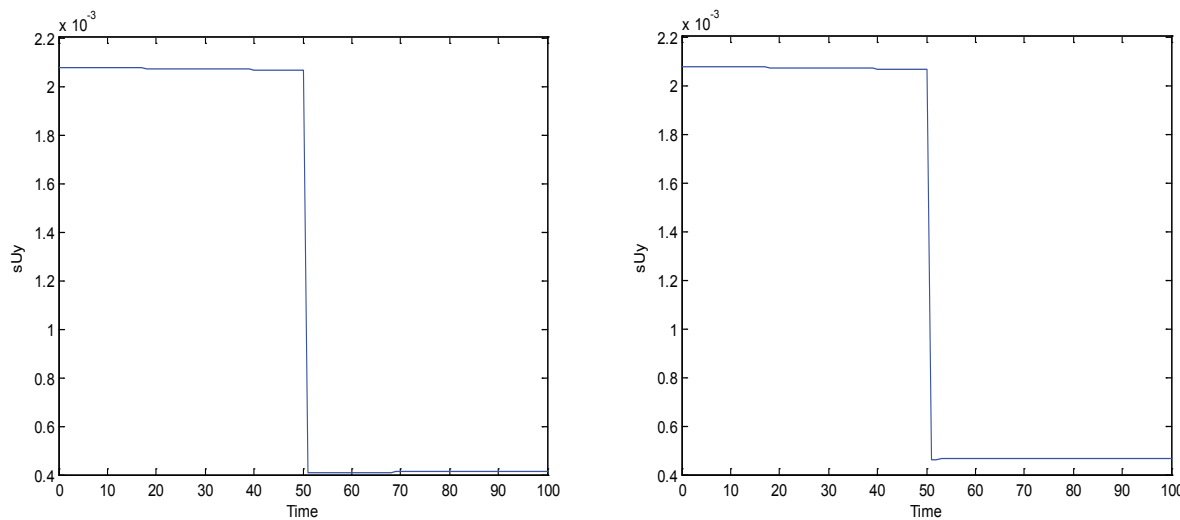

Gráfico 6 e 9

Taxa de crescimento do país U antes e depois da apreciação cambial no período 50 . Simulação 3
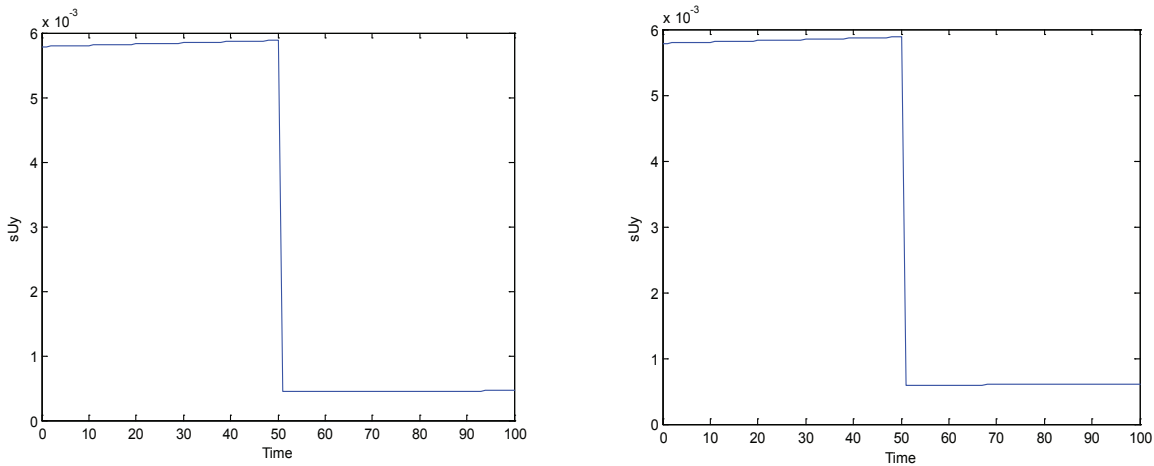